\title{
Treatment of mobile right heart thrombi with low-molecular-weight heparin
}

\author{
Konstantinos M Lampropoulos, Maria Bonou, Constantinos Theocharis, \\ John Barbetseas
}

Department of Cardiology, Polyclinic General Hospital of Athens, Athens, Greece

\section{Correspondence to}

Dr Konstantinos M Lampropoulos,

konlampropoulos@yahoo.gr

\section{SUMMARY}

Treatment of choice in patients with mobile thrombi in the right heart chambers is still controversial owing to the increased risk of recurrent pulmonary embolism (PE). Thrombolysis and surgical or catheter embolectomy are the preferred options. We present a case of an elderly patient with PE and free-floating atrial thrombi who was treated successfully with low-molecular-weight heparin (LMWH).

\section{BACKGROUND}

The visualisation of right-sided heart thrombi in patients with pulmonary embolism (PE) is below 4\%. A majority of them are in transit, arise from peripheral deep vein thrombosis and are associated with high death rate owing to recurrent PE. Thrombolysis, embolectomy or anticoagulation are the different options in the management of these patients. ${ }^{1}$

We report a case of a patient with PE and freefloating atrial thrombi who was treated successfully with low-molecular-weight heparin (LMWH).

\section{CASE PRESENTATION}

An 88-year-old man presented to our hospital after an episode of syncope. He had a 10-day history of inguinal surgery and was on bed rest during this period. Upon examination the patient was normotensive. The electrocardiogram demonstrated atrial fibrillation (AF) with a heart rate of $120 \mathrm{bpm}$. Oxygen saturation was $90 \%$ on room air. A bedside transthoracic echocardiogram visualised dilated right heart chambers, right ventricular hypokinesis and worm-like mobile masses in the right atrium consistent with free-floating thrombi (figure 1A,B). Laboratory tests showed elevated D-dimers $(>23100 \mu \mathrm{g} / \mathrm{l}$, normal values: 170-23000 $\mu \mathrm{g} / \mathrm{l})$.

The suspicion of PE as the cause of the syncope was evident and because of the increased risk of an acute massive new PE, we recommended thrombolysis or catheter embolectomy but the patient and his relatives denied. Thus therapeutic anticoagulation with LMWH, enoxaparin, was started. The patient also received diltiazem for heart rate control.

During hospitalisation there were no clinical or laboratory signs of repeat PE. Eighteen hours later, a new transthoracic echocardiographic examination showed the thrombus had been completely dissolved. 24 hours later spiral CT scanning which is the method of choice for the diagnosis of PE verified the existence of emboli in the right and left pulmonary arteries, while two residual thrombi $\left(1.61 \times 0.85\right.$ and $\left.0.972 \times 0.917 \mathrm{~cm}^{2}\right)$ in the left greater saphenous vein at the above-knee segment were imaged by ultrasonography (figure 1C-F). Acenocoumarol was added on the 5th day and LMWH was discontinued when international normalised ratio was $>2$. The patient was discharged after 12 days of hospitalisation in a good condition.

\section{TREATMENT}

According to the guidelines patients with mobile thrombi in the right heart chambers have an increased risk of recurrent PE; without treatment the death rate is $80-100 \%{ }^{1}$

The optimal therapy is still controversial. Thrombolysis and surgical or catheter embolectomy are the preferred options. Anticoagulation alone is less effective. No randomised trials exist comparing thrombolysis with embolectomy. Small studies show excellent results with lytic therapy, ${ }^{2}{ }^{3}$ on the contrary in the International Cooperative Pulmonary Embolism Registry (ICOPER Registry) the mortality was $>20 \% .^{4}$ The percentage of in situ thrombi lysis or their partial lysis, fragmentation and subsequent embolisation is also unknown. Unfractionated heparin (UFH) is not recommended because of poor results, while the use of LMWH has been validated in the treatment of right heart thrombi only in few case reports. $^{4-6}$

\section{OUTCOME AND FOLLOW-UP}

The use of LMWH instead of UFH was successful, as the patient improved, and at follow-up, 1 month later was free of symptoms.

\section{DISCUSSION}

Right heart thrombi may develop in situ in association with an underlying heart disease such as valvulopathy and AF, or may derive thrombi from peripheral venous clots migrating into the right heart. The latter are worm-like mobile thrombi mainly from deep and sometimes from superficial vein thrombosis of the lower limbs. ${ }^{1-3}$

The origin of the whole clinical picture in our patient was apparently the thrombosis of the left greater saphenous vein, while the coexistence of AF could have played a complementary role.

According to the guidelines, patients with mobile thrombi in the right heart chambers have increased risk of recurrent PE; without treatment the death rate is $80-100 \%$.

The optimal therapy is still controversial. Thrombolysis and surgical or catheter embolectomy are the preferred options. Anticoagulation alone is less effective. No randomised trials exist comparing thrombolysis with embolectomy. Small studies show excellent results with lytic 


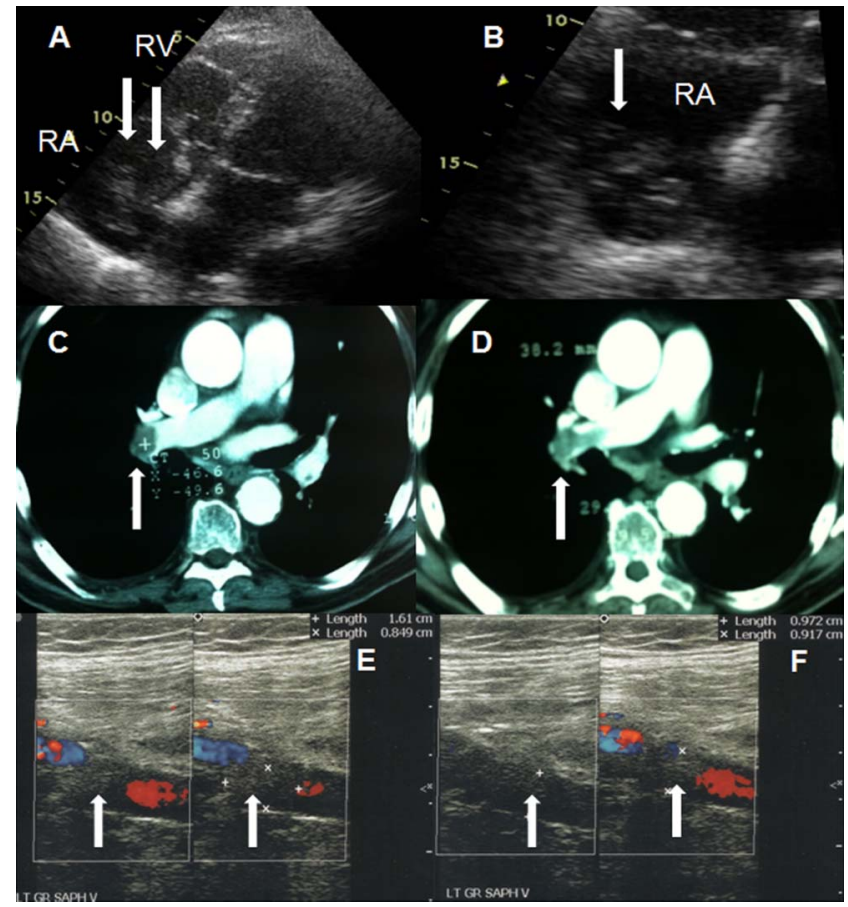

Figure 1 ( $A$ and $B$ ) A bedside transthoracic echocardiogram visualised dilated right heart chambers, right ventricular hypokinesis and worm-like mobile masses in the right atrium consistent with free-floating thrombi. (C and D) Spiral CT scanning verified the existence of pulmonary emboli in the right and left pulmonary artery. ( $E$ and F) B-mode and Doppler ultrasonography of the lower limbs demonstrated two residual thrombi diameter $(1.61 \times 0.85$ and $0.972 \times 0.917 \mathrm{~cm}^{2}$ ) in the left greater saphenous vein (prevein and postvein compression).

therapy ${ }^{4}$; on the contrary, in the ICOPER registry the mortality was $>20 \%$. $^{6}$ It is also unknown the percentage of in situ thrombi lysis or their partial lysis, fragmentation and subsequent embolisation. UFH is not recommended because of poor results, with overall death rates at 14 days, and at 3 and 70 months were $21 \%, 29 \%$ and $62.5 \%$, respectively, while the use of LMWH has been validated in the treatment of right heart thrombi only in few case reports. ${ }^{6-8}$ It is also well known that UFH and LMWH have no thrombolytic properties. They favour spontaneous fibrinolysis by the activated intrinsic fibrinolytic system, resulting in self-limitation or disappearance of the thrombus.

LMWHs have been proven to be no less safe and effective as intravenous UFH in the treatment of submassive PE. ${ }^{9}{ }^{10}$ In addition, LMWH monotherapy without oral anticoagulation appears promising and may be preferable in patients with malignancy. ${ }^{11}$ Advantages of LMWH include longer half-life, increased bioavailability, a more predictable dose response, they are dosed by weight, administered subcutaneously and usually do not require dose adjustments or laboratory monitoring. 1213

Our patient denied thrombolysis or catheter embolectomy. Cardiothoracic surgery was also excluded owing to his advanced age. The only available alternative therapy was anticoagulation with heparin. The use of LMWH instead of UFH was successful, as the patient improved and at follow-up, 1 month later was free of symptoms.

\section{Learning points}

Right heart thrombi may develop in situ in association with an underlying heart disease such as valvulopathy and atrial fibrillation or derive thrombi from peripheral venous clots migrating in the right heart. According to the guidelines patients with mobile thrombi in the right heart chambers have increased risk of recurrent pulmonary embolism (PE), without treatment the death rate is $80-100 \%$. ${ }^{1}$

- The optimal therapy is still controversial. Thrombolysis and surgical or catheter embolectomy are the preferred options. Anticoagulation alone is less effective. No randomised trials exist that compare thrombolysis with embolectomy. Small studies show excellent results with lytic therapy, ${ }^{45}$ on the contrary in the International Cooperative Pulmonary Embolism Registry the mortality was $>20 \%{ }^{6}$

- Unfractionated heparin (UFH) is not recommended because of poor results, with overall death rate at 14 days, at 3 and 70 months was $21 \%, 29 \%$ and $62.5 \%$, respectively, while the use of low-molecular-weight heparin (LMWH) has been validated in the treatment of right heart thrombi only in few case reports. $^{6-8}$

- LMWHs have been proven to be no less safe and effective as intravenous UFH in the treatment of submassive PE. ${ }^{9}{ }^{10}$ In addition, LMWH monotherapy without oral anticoagulation appears promising and may be preferable in patients with malignancy. ${ }^{11}$ Advantages of LMWH include longer half-life, increased bioavailability, a more predictable dose response, they are dosed by weight, administered subcutaneously and usually do not require dose adjustments or laboratory monitoring. ${ }^{12} 13$

\section{Competing interests None.}

\section{Patient consent Obtained.}

Provenance and peer review Not commissioned; externally peer reviewed.

\section{REFERENCES}

1 Torbicki A, Perrier A, Konstantinides S, et al. ESC Committee for Practice Guidelines (CPG). Guidelines on the diagnosis and management of acute pulmonary embolism: the task force for the diagnosis and management of acute pulmonary embolism of the European Society of Cardiology (ESC). Eur Heart J 2008;29:2276-315.

2 Verlato F, Zucchetta P, Prandoni P, et al. An unexpectedly high rate of pulmonary embolism in patients with superficial thrombophlebitis of the thigh. J Vasc Surg 1999:30:1113-15.

3 Sobreira ML, Maffei FH, Yoshida WB, et al. Prevalence of deep vein thrombosis and pulmonary embolism in superficial thrombophlebitis of the lower limbs: prospective study of 60 cases. Int Angiol 2009;28:400-8.

4 Kucher N, Rossi E, De Rosa M, et al. Massive pulmonary embolism. Circulation 2006;113:577-82.

5 Bonou M, Lampropoulos KM, Barbetseas J. Right heart free-floating thrombus after leg injury. BMJ Case Rep 2012;2012.doi:pii: bcr1220115366. 10.1136/bcr.12. 2011.5366

6 Torbicki A, Galie N, Covezzoli A, et al. Right heart thrombi in pulmonary embolism: results from the International Cooperative Pulmonary Embolism Registry. J Am Coll Cardiol 2003;41:2245-51.

7 Rose PS, Punjabi NM, Pearse DB. Treatment of right heart thromboemboli. Chest 2002;121:806-14.

8 Chartier L, Béra J, Delomez $M$, et al. Free-floating thrombi in the right heart: diagnosis, management, and prognostic indexes in 38 consecutive patients. Circulation 1999;99:2779-83.

9 Kucher N, Quiroz R, McKean S, et al. Extended enoxaparin monotherapy for acute symptomatic pulmonary embolism. Vasc Med 2005;10:251-6. 
10 Büller HR, Davidson BL, Decousus $\mathrm{H}$, et al. Matisse Investigators. Subcutaneous fondaparinux versus intravenous unfractionated heparin in the initial treatment of pulmonary embolism. N Engl J Med 2003;349:1695-702. Erratum in: N Engl J Med 2004;350:423.

11 Kakkar AK, Levine MN, Kadziola Z, et al. Low molecular weight heparin, therapy with dalteparin, and survival in advanced cancer: the Fragmin Advanced Malignancy Outcome Study (FAMOUS). J Clin Oncol 2004;22:1944-8.
12 Simonneau G, Sors H, Charbonnier B, et al. A comparison of low-molecular-weight heparin with unfractionated heparin for acute pulmonary embolism. The THESEE Study Group. Tinzaparine ou Heparine Standard: Evaluations dans I'Embolie Pulmonaire. N Engl J Med 1997;337:663-9.

13 Merli G, Spiro TE, Olsson CG, et al.; Enoxaparin Clinical Trial Group. Subcutaneous enoxaparin once or twice daily compared with intravenous unfractionated heparin for treatment of venous thromboembolic disease. Ann Intern Med 2001;134:191-202.

Copyright 2013 BMJ Publishing Group. All rights reserved. For permission to reuse any of this content visit http://group.bmj.com/group/rights-licensing/permissions.

BMJ Case Report Fellows may re-use this article for personal use and teaching without any further permission.

Become a Fellow of BMJ Case Reports today and you can:

- Submit as many cases as you like

- Enjoy fast sympathetic peer review and rapid publication of accepted articles

- Access all the published articles

- Re-use any of the published material for personal use and teaching without further permission

For information on Institutional Fellowships contact consortiasales@bmjgroup.com

Visit casereports.bmj.com for more articles like this and to become a Fellow 